\title{
The Snap Tension Analysis of Taut-Slack Mooring Line with tanh Method
}

\author{
Su-xia Zhang, Rui-zhi Shi, Shengli Chen, and Xi-jun Liu \\ School of Mechanical Engineering, Tianjin Key Laboratory of Nonlinear Dynamics \& Chaos Control, Tianjin University, \\ Tianjin 300072, China \\ Correspondence should be addressed to Su-xia Zhang; zhangsux@tju.edu.cn
}

Received 14 March 2017; Revised 6 June 2017; Accepted 24 July 2017; Published 29 August 2017

Academic Editor: Fazal M. Mahomed

Copyright (C) 2017 Su-xia Zhang et al. This is an open access article distributed under the Creative Commons Attribution License, which permits unrestricted use, distribution, and reproduction in any medium, provided the original work is properly cited.

\begin{abstract}
The continuous model is introduced, and the nonlinear partial differential equations of taut-slack mooring line system are transferred to nonlinear algebraic equations through tanh method, and four solitary solutions are obtained further. At the same time, to express the results clearly, the curve surfaces of strains, displacements, and tension are plotted. The results show that there are four different solutions in the system. With the pretension increasing, the tension changes from one solitary solution to snap tension, and when the pretension is increased further, the curve converts to continuous line, until straight line, which is corresponding to the taut mooring line. In the process of increasing of pretension, the mooring line transfers from slack to taut, accompanied with tension skipping, which is reduced by the system parameters, and different combination of parameters may introduce different tension in line, and the uncertainty may cause the breakage of mooring system. The results have an agreement with experiment, which shows that the calculating method in this paper may be believable and feasible. This work may provide reference for design of mooring system.
\end{abstract}

\section{Introduction}

With the development of exploitation of deep-water resources, TLP and SPAR platforms are designed and used in some areas and are considered as the excellent platforms in deep water, in which mooring line is one of the most important parts of platforms.

Mooring lines are flexible structures and have unidirectional stiffness; that is, they can only bear tension and cannot bear compressive loading. When the platform moves with large amplitude, the mooring line will transfer from taut to slack, accompanied with snap tension. The research showed that the snap tension was usually several times to dozen times more than the mean tension [1], which may be the real reason causing breakage of mooring line.

Many works have been done by the scholars to reveal the mechanism of breakage of mooring line. Niedzwecki and Thampi [2] investigated the snap load behavior of marine cable systems in regular seas. Huang and Vassalos studied the impulse loads under alternative taut-slack conditions by using the model of bilinear string [3,4]. They neglected the effect of floating structure, the cable was simplified into the model subjected to periodic sinusoidal excitation at the top end of cable, and the snap tension of the mooring cable was calculated [5]. The results show that the effect of tension magnification is due to the shock. Experiments show that, under sinusoidal excitation and quasi-static condition, the result calculated by the quasi-static method is near the experiment result. But under the impact condition, the impact tension is much larger than the result calculated by the quasi-static method [6]. The equivalent force model is used to analyze the effect of mooring systems on the horizontal motions [7]. Zhang et al. studied the analytical solution of taut-slack mooring line with tanh method and obtained snap tension curves in four cases [8]. Until now, most of the solutions to the equations are numerical simulation, and the analytical solution is not fully developed.

At the same time, different methods solving the nonlinear evolution equations have been developed. The tanh method, developed for years, is one of the most direct and effective algebraic methods for finding exact solutions of nonlinear diffusion equations. Recently, much work has been concentrated on the various extension and applications of the method [9]. Huibin and Kelin [10] introduced a 
power series in tanh as a possible solution and substituted this expansion directly into a higher-order $\mathrm{KdV}$ equation. Wazwaz [11] employed the tanh method for traveling wave solutions of nonlinear equations and the work was extended to equations that do not have tanh polynomial solutions. Abdou and Soliman [12] presented and implemented the solutions of nonlinear physical equations for constructing multiple traveling wave in a computer algebraic system by means of computerized symbolic computation and a modified extended tanh-function method. Zheng et al. [13] presented the generalized extended tanh-function method for constructing the exact solutions of nonlinear partial differential equations (NPDEs) in a unified way making use of a new generalized analysis. As a result, the solitary wave solutions and other new and more general solutions were obtained. Based on the symbolic computation, Zhi and Zhang [14] combined the tanh-function method with the symmetry method to construct new type of solutions of the nonlinear evolution equations for the first time. With the combined method, some new types of solutions of the coupled $(2+1)$ dimensional nonlinear system of Schrodinger equations were obtained.

In this paper, to reveal the mechanism of snap tension in mooring line while transforming from taut to slack or from slack to taut, the continuous model is introduced and tanh method is used to resolve the nonlinear equations of tautslack mooring line. Four types of analytical solutions of tautslack mooring line are obtained, and the displacement and tension curve surfaces in different cases are presented, and the effects of pretension will be discussed in Section 4 . The snap tension is obtained, which may be the reason causing the breakage of mooring line.

\section{The tanh Method}

Consider a given evolution, say in two variables [15],

$$
H\left(u, u_{t}, u_{x}, u_{x x}, \ldots\right)=0,
$$

where $u=u(x, t)$ and $H$ is a polynomial about $u$ and its derivatives.

The fact that the solutions of many nonlinear equations can be expressed as a finite series of tanh function motivates us to seek for the solutions of (1) in the form

$$
u(x, t)=u(\xi)=\sum_{i=0}^{m} a_{i} T^{i}=\sum_{i=0}^{m} a_{i} \tanh (\xi)^{i},
$$

where $\xi=\kappa(x-c t)+\xi_{0}$. Notice that the highest order of $\mathbf{d}^{p} u / \mathbf{d} \xi^{p}$ is

$$
\begin{aligned}
o\left(\frac{\mathbf{d}^{p} u}{\mathbf{d} \xi^{p}}\right) & =m+p, \quad p=1,2,3, \ldots, \\
o\left(u^{q} \frac{\mathbf{d}^{p} u}{\mathbf{d} \xi^{p}}\right) & =(q+1) m+p, \\
q & =1,2,3, \ldots ; p=1,2,3, \ldots
\end{aligned}
$$

So $m$ can be obtained by balancing the derivative term of the highest order with the nonlinear term in (1). $k, c, a_{0}, \ldots, a_{m}$ are parameters to be determined. Substituting (2) into (1) will yield a set of algebraic equations for $k, c, a_{0}, \ldots, a_{m}$ because all coefficients of $\tanh (\xi)^{i}$ have to vanish. Using these relations, $k, c, a_{0}, \ldots, a_{m}$ can be obtained. Therefore, the traveling solitary wave solutions are obtained. Usually $m$ is a positive integer; however, once in a while, the value of $m$ is a negative or a fraction. In these cases, we can introduce a transformation $u(\xi)=v(\xi)^{\operatorname{sign}(m) / d}$, where $d$ is the denominator of $m$, and transform (1) into another equation for $v$, whose balancing number will be a positive integer. Then it can be dealt with by the above method. Therefore the final solution $u$ may be a rational or radical function about $\tanh (\xi)$.

\section{Equations of Motion}

The model of mooring line is shown in Figure 1. The horizontal uniform mooring line is considered, and the shear deformation is neglected. The left end of the line is fixed, and the right end is attached to the buoy; here the condition is simplified by assuming harmonic excitation $A_{b} \sin \omega t$. $e_{1}$ and $e_{2}$ are the unit vector in tangential and normal direction, respectively, and $e_{3}$ is the binormal unit vector. $\chi^{o}$ and $\chi^{f}$ represent the static and dynamic configuration, and $U\left(U_{1}, U_{2}, U_{3}\right)$ is the displacement relative to the static configuration.

Only the in-plane motions of mooring line are considered, and the equations are as follows [16]:

$$
\begin{aligned}
\rho A U_{1, t t}= & {\left[(P+E A \varepsilon)\left(1+U_{1, s}-\psi U_{2}\right)\right]_{, s} } \\
& -\psi(P+E A \varepsilon)\left(U_{2, S}+\psi U_{1}\right) \\
& -\left(\rho-\rho_{w}\right) A g l_{t}+F_{1} \\
\rho A U_{2, t t}= & {\left[(P+E A \varepsilon)\left(U_{2, S}+\psi U_{1}\right)\right]_{, s} } \\
& +\psi(P+E A \varepsilon)\left(1+U_{1, s}-\psi U_{2}\right) \\
& -\left(\rho-\rho_{w}\right) A g l_{n}+F_{2},
\end{aligned}
$$

where $\varepsilon$ is the dynamic strain, and the expression is as follows:

$$
\varepsilon=U_{1, s}-\psi U_{2}+\frac{1}{2}\left[\left(U_{1, s}-\psi U_{2}\right)^{2}+\left(U_{2, s}+\psi U_{1}\right)^{2}\right] .
$$

$E A$ represents the cable section modulus, $\rho A$ represents the cable mass/length, and $F_{1}, F_{2}$ are components of an arbitrary external excitation, including the hydrodynamic loading and exciting from the buoy attached at the end, mainly the end exciting. In other words, the transforming from taut to slack of mooring line is mainly caused by the large amplitude motion of attached buoy. The hydrodynamic loading is calculated with Morison's equation as follows:

$$
\begin{aligned}
& F_{1}=C_{a t} \rho_{w} \frac{\pi d^{2}}{4} U_{1, t t}+C_{d t} \frac{\rho_{w} d}{2}\left|U_{1, t}-v_{t}\right|\left(U_{1, t}-v_{t}\right) \\
& F_{2}=C_{a n} \rho_{w} \frac{\pi d^{2}}{4} U_{2, t t}+C_{d n} \frac{\rho_{w} d}{2}\left|U_{2, t}-v_{n}\right|\left(U_{2, t}-v_{n}\right) .
\end{aligned}
$$

$C_{a n}$ and $C_{a t}$ are the added mass coefficients in tangential and normal direction, respectively, $C_{d t}$ and $C_{d n}$ are fluid drag 


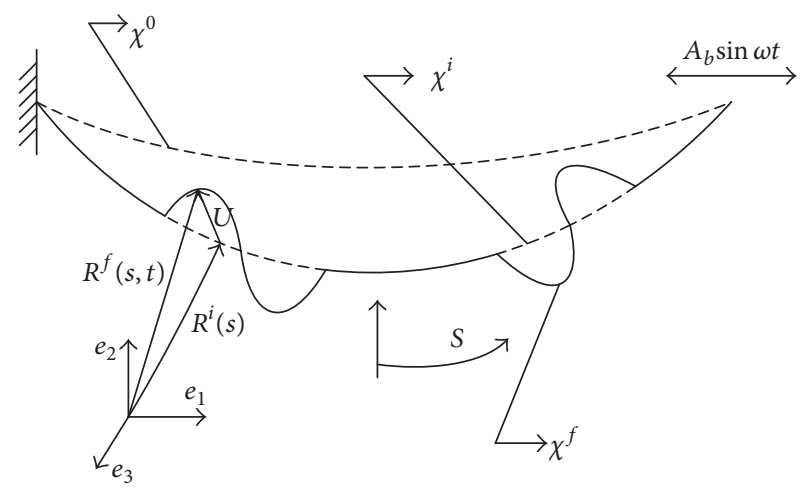

(a)

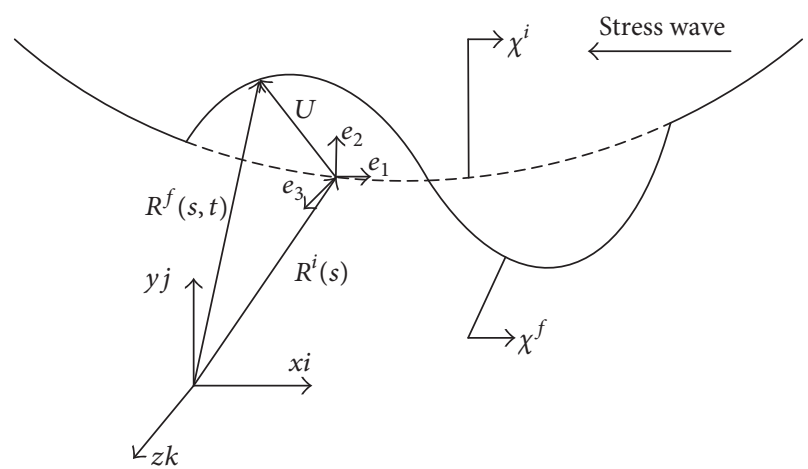

(b)

FIGURE 1: The model of mooring line.

coefficients in tangential and normal direction, respectively, $v$ is the velocity of fluid particle, and the subscripts $t$ and $n$ denote the projection in tangential and normal directions, respectively. $U_{1}$ and $U_{2}$ are the displacements in tangential and normal directions, subscript $s$ denotes partial differentiation with respect to arc lengths, and $t$ denotes partial differentiation with respect to time. Moreover, $P(s)$ and $\psi(s)$ are the equilibrium tension and curvature distributions describing a catenary as given by

$$
\begin{aligned}
& P=\sqrt{P_{0}^{2}+(\rho A g s)^{2}}, \\
& \psi=\frac{\rho A g P_{0}}{\left(P_{0}^{2}+(\rho A g s)^{2}\right)},
\end{aligned}
$$

where $P_{0}$ denotes the horizontal component of the equilibrium cable tension.

Now considering the effects of curvature and tension in line, substitute (6) and (8) into (4) and (5), and the equations can be reduced as

$$
\begin{aligned}
& g\left(\frac{3}{2} \lambda^{3} s^{2}-\frac{1}{2} \lambda^{7} s^{6}-\lambda\right) U_{1}+\left(g \lambda^{2} s+2 g \lambda^{4} s^{3}\right. \\
& \left.+2 \frac{E}{\rho} \lambda^{3} s\right) U_{2}+g \lambda s U_{1, s}+2 \frac{E}{\rho}\left(\lambda^{6} s^{3}-\lambda^{4} s\right) U_{1}^{2} \\
& +\left(g \lambda^{2} s^{2}+\frac{E}{\rho} \lambda^{3} s^{2}+g \lambda^{4} s^{4}-\frac{E}{\rho} \lambda-2 g\right) U_{2, s}+6 \\
& \quad+\frac{E}{\rho}\left(\lambda^{6} s^{3}-\lambda^{4} s\right) U_{2}^{2}+\frac{E}{\rho}\left(\lambda^{3} s^{2}-\lambda\right) U_{2} U_{2, s} U_{2, s s} \\
& +\frac{E}{\rho}\left(2 \lambda^{6} s^{2}-3 \lambda^{8} s^{4}+2 \lambda^{10} s^{6}-\frac{1}{2} \lambda^{12} s^{8}-\frac{1}{2} \lambda^{4}\right) U_{1}^{3} \\
& +3 \frac{E}{\rho}\left(\lambda^{5} s-2 \lambda^{7} s^{3}+\lambda^{9} s^{5}\right) U_{2}^{3}+\left(\frac{E}{\rho}+\frac{P_{0}}{\rho A}\right. \\
& \left.+\frac{1}{2} g \lambda s^{2}\right) U_{1, s s}-\left(1+C_{a t} \rho_{w} \frac{\pi d^{2}}{4 \rho A}\right) U_{1, t t}+C_{d t}
\end{aligned}
$$

$$
\begin{aligned}
& \cdot \frac{\rho_{w} d}{2 \rho A}\left|U_{1, t}-v_{t}\right|\left(U_{1, t}-v_{t}\right)+g \lambda s+3 \frac{E}{\rho} U_{1, s} U_{1, s s} \\
& +\frac{E}{\rho} U_{2, s} U_{2, s s}+\frac{3}{2} \frac{E}{\rho} U_{1, s}^{2} U_{1, s s}+4 \frac{E}{\rho}\left(\lambda^{2}-2 \lambda^{4} s^{2}\right. \\
& \left.+\lambda^{6} s^{4}\right) U_{2} U_{2, s}+\frac{E}{\rho}\left(\lambda^{3} s^{2}-\lambda\right) U+2 \frac{E}{\rho}\left(3 \lambda^{5} s^{2}\right. \\
& \left.-3 \lambda^{7} s^{4}+\lambda^{9} s^{6}-\lambda^{3}\right) U_{2}^{2} U_{2, s}+2 \frac{E}{\rho}\left(\lambda^{6} s^{3}-\lambda^{4} s\right) \\
& \cdot U_{1}^{2} U_{1, s}+\frac{E}{\rho} \lambda^{3} s U_{2, s}^{2} U_{22, s}^{3}+\frac{E}{\rho}\left(\frac{1}{2} \lambda^{6} s^{4}+\frac{1}{2} \lambda^{2}\right. \\
& \left.-\lambda^{4} s^{2}\right) U_{1, s}^{2} U_{1}+\frac{E}{\rho}\left(\frac{1}{2} \lambda^{2}-\lambda^{4} s^{2}+\frac{1}{2} \lambda^{6} s^{4}\right) U_{1}^{2} U_{1, s s} \\
& =0 \\
& g\left(1-\lambda^{4} s^{4}-\lambda^{2} s^{2}\right)+g\left(-\lambda^{2} s-2 \lambda^{4} s^{3}\right) U_{1}+g \lambda s U_{2, s} \\
& +\frac{5}{2} \frac{E}{\rho}\left(\lambda-\lambda^{3} s^{2}\right) U_{1, s}^{2}-\left(1+C_{a n} \rho_{w} \frac{\pi d^{2}}{4 \rho A}\right) U_{2, t t} \\
& +C_{d n} \frac{\rho_{w} d}{2 \rho A}\left|U_{2, t}-v_{n}\right|\left(U_{2, t}-v_{n}\right)+\left(2 \frac{E}{\rho} \lambda^{4} s^{2}\right. \\
& \left.-\frac{E}{\rho} \lambda^{6} s^{4}-g \lambda-\frac{1}{2} g \lambda^{7} s^{6}+\frac{3}{2} g \lambda^{3} s^{2}-\frac{E}{\rho} \lambda^{2}\right) U_{2}+3 \\
& \cdot \frac{E}{\rho}\left(-\lambda^{5} s+2 \lambda^{7} s^{3}-\lambda^{9} s^{5}\right) U_{1}^{3}+\frac{E}{\rho}\left(2 \lambda^{6} s^{2}-3 \lambda^{8} s^{4}\right. \\
& \left.+2 \lambda^{10} s^{6}-\frac{1}{2} \lambda^{12} s^{8}-\frac{1}{2} \lambda^{4}\right) U_{2}^{3}+\left(2 g-\frac{E}{\rho} \lambda^{3} s^{2}\right. \\
& \left.-g \lambda^{4} s^{4}-g \lambda^{2} s^{2}+\frac{E}{\rho} \lambda\right) U_{1, s}+\frac{1}{2} \frac{E}{\rho}\left(3 \lambda^{7} s^{4}-\lambda^{9} s^{6}\right. \\
& \left.-3 \lambda^{5} s^{2}+\lambda^{3}\right) U_{1}^{2}+\frac{3}{2} \frac{E}{\rho}\left(3 \lambda^{7} s^{4}-\lambda^{9} s^{6}-3 \lambda^{5} s^{2}\right.
\end{aligned}
$$




$$
\begin{aligned}
& \left.+\lambda^{3}\right) U_{2}^{2}+\left(\frac{1}{2} g \lambda s^{2}+\frac{P_{0}}{\rho A}\right) U_{2, s s}+\frac{1}{2} \frac{E}{\rho}\left(\lambda^{3} s^{2}-\lambda\right) \\
& \cdot U_{2, s}^{2}+\frac{E}{\rho}\left(\lambda-\lambda^{3} s^{2}\right) U_{1, s}^{3}+2 \frac{E}{\rho} \lambda^{3} s U_{2} U_{2, s}+2 \\
& \cdot \frac{E}{\rho}\left(3 \lambda^{7} s^{4}-3 \lambda^{5} s^{2}-\lambda^{9} s^{6}+\lambda^{3}\right) U_{1, s} U_{1}^{2}+2 \frac{E}{\rho}\left(\lambda^{6} s^{3}\right. \\
& \left.-\lambda^{4} s\right) U_{2, s} U_{2}^{2}-\frac{E}{\rho} \lambda^{3} s U_{1} U_{1, s}^{2}+\frac{E}{\rho}\left(\lambda^{3} s^{2}-\lambda\right) U_{2} U_{2, s s} \\
& +\frac{E}{\rho}\left(\lambda-\lambda^{3} s^{2}\right) U_{1} U_{1, s} U_{1, s s}+\frac{E}{\rho}\left(\frac{1}{2} \lambda^{6} s^{4}-\lambda^{4} s^{2}\right. \\
& \left.+\frac{1}{2} \lambda^{2}\right) U_{2} U_{2, s}^{2}+\frac{3}{2} \frac{E}{\rho} U_{2, s}^{2} U_{2, s s}+\frac{E}{\rho}\left(\frac{1}{2} \lambda^{2}-\lambda^{4} s^{2}\right. \\
& \left.+\frac{1}{2} \lambda^{6} s^{4}\right) U_{2}^{2} U_{2, s s}+\frac{E}{\rho}\left(\lambda-\lambda^{3} s^{2}\right) U_{1} U_{1, s s}-2 \frac{E}{\rho} \\
& \cdot \lambda^{3} s U_{1} U_{1, s}=0,
\end{aligned}
$$

where $\lambda=\rho A g / P_{0}$. The dynamic tension $T$ is that

$$
T=P_{0}+E A \varepsilon
$$

In the quasi-static fluid, $v_{t}=0$ and $v_{n}=0$. Then the initial equations may be simplified and rewritten as follows:

$$
\begin{aligned}
& A_{1} U_{1, s s}-A_{2} U_{1, t t}+A_{3}+A_{4} U_{1}+A_{5} U_{2}+A_{6} U_{1, s} \\
& +A_{7} U_{2, s}+A_{8} U_{1}^{2}+A_{9} U_{2}^{2}+A_{10} U_{1}^{3}+A_{11} U_{2}^{3} \\
& +A_{12} U_{1, s} U_{1, s s}+A_{13} U_{2, s} U_{2, s s}+A_{14} U_{1, s}^{2} U_{1, s s} \\
& +A_{15} U_{2} U_{2, s}+A_{16} U_{2}^{2} U_{2, s}+A_{17} U_{1}^{2} U_{1, s} \\
& +A_{18} U_{1, s}^{2} U_{1}+A_{19} U_{2, s}^{2} U_{2}+A_{20} U_{2, s}^{3} \\
& +A_{21} U_{2} U_{2, s} U_{2, s s}+A_{22} U_{1}^{2} U_{1, s s} \\
& +A_{23} \operatorname{sgn}\left(U_{1, t}\right) U_{1, t}^{2}=0 \\
& B_{1} U_{2, s s}-B_{2} U_{2, t t}+B_{3}+B_{4} U_{1}+B_{5} U_{2}+B_{6} U_{1, s} \\
& +B_{7} U_{2, s}+B_{8} U_{1}^{2}+B_{9} U_{2}^{2}+B_{10} U_{1}^{3}+B_{11} U_{2}^{3} \\
& +B_{12} U_{1, s}^{2}+B_{13} U_{2, s}^{2}+B_{14} U_{1, s}^{3}+B_{15} U_{2} U_{2, s} \\
& +B_{16} U_{1} U_{1, s}+B_{17} U_{1, s} U_{1}^{2}+B_{18} U_{2, s} U_{2}^{2}-B_{19} U_{1} U_{1, s}^{2} \\
& +B_{20} U_{2} U_{2, s}^{2}+B_{21} U_{1} U_{1, s s}+B_{22} U_{2} U_{2, s s} \\
& +B_{23} U_{1} U_{1, s} U_{1, s s}+B_{24} U_{2, s}^{2} U_{2, s s}+B_{25} U_{2}^{2} U_{2, s s} \\
& +B_{26} \operatorname{sgn}\left(U_{2, t}\right) U_{2, t}^{2}=0 \\
& +x_{1}
\end{aligned}
$$

where $A_{i}$ and $B_{i}$ are coefficients, shown in the Appendix.

\section{Results and Discussions}

Assuming that $U_{1}, U_{2}$ are the polynomials of $T$ with $m$ and $n$ ranks, respectively, the nonlinear term $A_{14} \kappa^{4}\left(U_{1}^{\prime}\right)^{2} U_{1}^{\prime \prime}$ in (11) is $3 m+4$-order polynomials of $T$, and $A_{21} \kappa^{3} U_{2} U_{2}^{\prime} U_{2}^{\prime \prime}$ is $3 n+3$-order polynomials of $T$, while the highest order linear term is $A_{1} \kappa^{2} U_{1}^{\prime \prime}$, which is $m+2$ polynomials of $T$. Similarly, in (12), the nonlinear terms $B_{23} \kappa^{3} U_{1} U_{1}^{\prime} U_{1}^{\prime \prime}$ and $B_{24} \kappa^{4}\left(U_{2}^{\prime}\right)^{2} U_{2}^{\prime \prime}$ are $3 m+3$ and $3 n+4$ polynomials of $T$, respectively, and the highest order linear term $B_{1} \kappa^{2} U_{2}^{\prime \prime} n+2$. To look for the traveling wave solution of (11) and (12), the parameters $m$ and $n$ are first determined by balancing the linear term of highest order with the nonlinear term:

$$
\begin{aligned}
& \max (3 m+4,3 n+3)=m+2 \\
& \max (3 n+4,3 m+3)=n+2 .
\end{aligned}
$$

It is solved that
(1) $\left\{\begin{array}{l}m=-1 \\ n=-1\end{array}\right.$
(2) $\left\{\begin{array}{l}m=-1 \\ n=-2\end{array}\right.$
(3) $\left\{\begin{array}{l}m=-2 \\ n=-1\end{array}\right.$
(4) $\left\{\begin{array}{l}m=-\frac{1}{2} \\ n=-\frac{1}{2} .\end{array}\right.$

For cases (2), (3), and (4), there is no nontrivial solution in terms of tanh function. For case (1), the following transformation is introduced:

$$
\begin{aligned}
& U_{1}(\xi)=\frac{1}{b_{0}+b_{1} \tanh (\xi)}, \\
& U_{2}(\xi)=\frac{1}{a_{0}+a_{1} \tanh (\xi)}, \\
& \xi=\kappa\left(x-c_{1} t\right),
\end{aligned}
$$

where $a_{0}, a_{1}, b_{0}, b_{1}, \kappa$, and $c_{1}$ are undetermined coefficients.

Submitting the transformation into (9), collecting the coefficients of $T$ with the same orders, the nonlinear algebraic equations can be obtained:

$$
\begin{aligned}
P_{1}= & A_{5} b_{0}+A_{3}+A_{9} b_{0}^{2}+A_{4} a_{0}+A_{15} \kappa b_{1} b_{0} \\
& +A_{16} \kappa b_{1} b_{0}^{2}+A_{8} a_{0}^{2}+A_{6} \kappa a_{1}+A_{10} a_{0}^{3}+A_{11} b_{0}^{3} \\
& +A_{18} \kappa^{2} a_{0} a_{1}^{2}+A_{17} \kappa a_{1} a_{0}^{2}+A_{19} \kappa^{2} b_{0} b_{1}^{2}+A_{7} \kappa b_{1} \\
& +A_{20} \kappa^{3} b_{1}^{3}=0
\end{aligned}
$$




$$
\begin{aligned}
P_{2}= & 2 A_{8} a_{1} a_{0}+2 A_{9} b_{1} b_{0}+3 A_{10} a_{1} a_{0}^{2}+A_{4} a_{1}+A_{5} b_{1} \\
& +3 A_{11} b_{1} b_{0}^{2}+A_{15} \kappa b_{1}^{2}+A_{18} \kappa^{2} a_{1}^{3}+A_{19} \kappa^{2} b_{1}^{3} \\
& +2 A_{16} \kappa b_{0} b_{1}^{2}+2 A_{17} \kappa a_{0} a_{1}^{2}=0 \\
P_{3}= & 3 A_{10} a_{0} a_{1}^{2}+A_{8} a_{1}^{2}+A_{9} b_{1}^{2}+3 A_{11} b_{0} b_{1}^{2}+A_{16} \kappa b_{1}^{3} \\
& +A_{17} \kappa a_{1}^{3}=0 \\
P_{4}= & A_{11} b_{1}^{3}+A_{10} a_{1}^{3}=0 \\
P_{5}= & B_{7} \kappa b_{1}+B_{12} \kappa^{2} a_{1}^{2}-B_{19} \kappa^{2} a_{0} a_{1}^{2}+B_{6} \kappa a_{1}+B_{4} a_{0} \\
& +B_{8} a_{0}^{2}+B_{9} b_{0}^{2}+B_{10} a_{0}^{3}+B_{3}+B_{11} b_{0}^{3}+B_{5} b_{0} \\
& +B_{13} \kappa^{2} b_{1}^{2}+B_{15} \kappa b_{1} b_{0}+B_{18} \kappa b_{1} b_{0}^{2}+B_{14} \kappa^{3} a_{1}^{3}
\end{aligned}
$$

$$
\begin{aligned}
& -B_{16} \kappa a_{0} a_{1}+B_{17} \kappa a_{1} a_{0}^{2}+B_{20} \kappa^{2} b_{1}^{2} b_{0}=0 \\
P_{6}= & 2 B_{8} a_{1} a_{0}+2 B_{9} b_{1} b_{0}+3 B_{10} a_{1} a_{0}^{2}+B_{4} a_{1}+B_{5} b_{1} \\
& +3 B_{11} b_{1} b_{0}^{2}+B_{15} \kappa b_{1}^{2}+2 B_{18} \kappa b_{1}^{2} b_{0}+B_{20} \kappa^{2} b_{1}^{3} \\
& -B_{19} \kappa^{2} a_{1}^{3}=0 \\
P_{7}= & 3 B_{10} a_{0} a_{1}^{2}+B_{8} a_{1}^{2}+B_{9} b_{1}^{2}+3 B_{11} b_{0} b_{1}^{2}+B_{18} \kappa b_{1}^{3} \\
& +B_{17} \kappa a_{1}^{3}=0 \\
P_{8}= & B_{11} b_{1}^{3}+B_{10} a_{1}^{3}=0 .
\end{aligned}
$$

Solving them with RATH package $[17,18]$, the four solutions are obtained.

Case 1. The parameters are that

$$
\begin{aligned}
c_{1}= & -\frac{\sqrt{B_{1} B_{2}}}{B_{2}} \\
\kappa= & \frac{\sqrt{\left(-B_{4} A_{9}+A_{4} B_{9}\right)\left(-4 A_{4} B_{3} A_{9}^{2}+4 A_{3} B_{4} A_{9}^{2}-A_{9} B_{4} A_{5}^{2}+B_{9} A_{4} A_{5}^{2}\right)} B_{9}}{2\left(-B_{4} A_{9}+A_{4} B_{9}\right) B_{7} A_{9}} \\
a_{0}= & -\frac{A_{5}}{2 A_{9}} \\
a_{1}= & \frac{\sqrt{\left(-B_{4} A_{9}+A_{4} B_{9}\right)\left(-4 A_{4} B_{3} A_{9}^{2}+4 A_{3} B_{4} A_{9}^{2}-A_{9} B_{4} A_{5}^{2}+B_{9} A_{4} A_{5}^{2}\right)}}{2\left(-B_{4} A_{9}+A_{4} B_{9}\right) A_{9}} \\
b_{0}= & \frac{-B_{4} A_{9}+A_{4} B_{9}}{2\left(-B_{3} A_{9}+A_{3} B_{9}\right)} \\
b_{1}= & -A_{6} \sqrt{\left(-B_{4} A_{9}+A_{4} B_{9}\right)\left(-4 A_{4} B_{3} A_{9}^{2}+4 A_{3} B_{4} A_{9}^{2}-A_{9} B_{4} A_{5}^{2}+B_{9} A_{4} A_{5}^{2}\right)} \\
2\left(-B_{3} A_{9}+A_{3} B_{9}\right) B_{7} A_{4} A_{9} & B_{9} .
\end{aligned}
$$

The constraint parameters are that

$$
\begin{aligned}
& B_{8}=-\frac{B_{4}\left(-4 A_{6}^{2} B_{9}^{2} A_{9}^{2} A_{4} B_{3}+4 A_{6}^{2} B_{9}^{2} A_{9}^{2} A_{3} B_{4}-A_{6}^{2} B_{9}^{2} A_{5}^{2} B_{4} A_{9}+A_{6}^{2} B_{9}^{3} A_{5}^{2} A_{4}+A_{4}^{2} A_{9}^{3} B_{7}^{2} B_{4}-A_{4}^{3} A_{9}^{2} B_{7}^{2} B_{9}\right)}{4 A_{4}^{2} A_{9}^{2} B_{7}^{2}\left(B_{9} A_{3}-A_{9} B_{3}\right)} \\
& A_{7}=\frac{A_{9} B_{7}}{B_{9}}, \\
& B_{5}=\frac{A_{5} B_{9}}{A_{9}}, \\
& A_{8}=-\frac{-4 A_{6}^{2} B_{9}^{2} A_{9}^{2} A_{4} B_{3}+4 A_{6}^{2} B_{9}^{2} A_{9}^{2} A_{3} B_{4}-A_{6}^{2} B_{9}^{2} A_{5}^{2} B_{4} A_{9}+A_{6}^{2} B_{9}^{3} A_{5}^{2} A_{4}+A_{4}^{2} A_{9}^{3} B_{7}^{2} B_{4}-A_{4}^{3} A_{9}^{2} B_{7}^{2} B_{9}}{4 A_{4} A_{9}^{2} B_{7}^{2}\left(B_{9} A_{3}-A_{9} B_{3}\right)},
\end{aligned}
$$




$$
\begin{aligned}
& B_{6}=\frac{A_{6} B_{4}}{A_{4}}, \\
& A_{1}=\frac{A_{2} B_{1}}{B_{2}} .
\end{aligned}
$$

And other parameters are arbitrary.

Case 2. The parameters are that

$$
\begin{aligned}
& c_{1}=\frac{\sqrt{B_{1} B_{2}}}{B_{2}}, \\
& a_{0}=-\frac{A_{5}}{2 A_{9}}, \\
& a_{1}=-\frac{\sqrt{\left(-B_{4} A_{9}+A_{4} B_{9}\right)\left(-4 A_{4} B_{3} A_{9}^{2}+4 A_{3} B_{4} A_{9}^{2}-A_{9} B_{4} A_{5}^{2}+B_{9} A_{4} A_{5}^{2}\right)}}{2\left(-B_{4} A_{9}+A_{4} B_{9}\right) A_{9}} \\
& b_{1}=\frac{A_{6} \sqrt{\left(-B_{4} A_{9}+A_{4} B_{9}\right)\left(-4 A_{4} B_{3} A_{9}^{2}+4 A_{3} B_{4} A_{9}^{2}-A_{9} B_{4} A_{5}^{2}+B_{9} A_{4} A_{5}^{2}\right)}}{2\left(-B_{3} A_{9}+A_{3} B_{9}\right) B_{7} A_{4} A_{9}} \\
& b_{0}=-\frac{-B_{4} A_{9}+A_{4} B_{9}}{2\left(-B_{3} A_{9}+A_{3} B_{9}\right)}, \\
& \kappa=-\frac{\sqrt{\left(-B_{4} A_{9}+A_{4} B_{9}\right)\left(-4 A_{4} B_{3} A_{9}^{2}+4 A_{3} B_{4} A_{9}^{2}-A_{9} B_{4} A_{5}^{2}+B_{9} A_{4} A_{5}^{2}\right)} B_{9}}{2\left(-B_{4} A_{9}+A_{4} B_{9}\right) B_{7} A_{9}} .
\end{aligned}
$$

The constraint parameters are that

$$
\begin{aligned}
& B_{8}=-\frac{B_{4}\left(-4 A_{6}^{2} B_{9}^{2} A_{9}^{2} A_{4} B_{3}+4 A_{6}^{2} B_{9}^{2} A_{9}^{2} A_{3} B_{4}-A_{6}^{2} B_{9}^{2} A_{5}^{2} B_{4} A_{9}+A_{6}^{2} B_{9}^{3} A_{5}^{2} A_{4}+A_{4}^{2} A_{9}^{3} B_{7}^{2} B_{4}-A_{4}^{3} A_{9}^{2} B_{7}^{2} B_{9}\right)}{4 A_{4}^{2} A_{9}^{2} B_{7}^{2}\left(B_{9} A_{3}-A_{9} B_{3}\right)} \\
& A_{7}=\frac{A_{9} B_{7}}{B_{9}}, \\
& B_{5}=\frac{A_{5} B_{9}}{A_{9}} \\
& A_{8}=-\frac{-4 A_{6}^{2} B_{9}^{2} A_{9}^{2} A_{4} B_{3}+4 A_{6}^{2} B_{9}^{2} A_{9}^{2} A_{3} B_{4}-A_{6}^{2} B_{9}^{2} A_{5}^{2} B_{4} A_{9}+A_{6}^{2} B_{9}^{3} A_{5}^{2} A_{4}+A_{4}^{2} A_{9}^{3} B_{7}^{2} B_{4}-A_{4}^{3} A_{9}^{2} B_{7}^{2} B_{9}}{4 A_{4} A_{9}^{2} B_{7}^{2}\left(B_{9} A_{3}-A_{9} B_{3}\right)} \\
& B_{6}=\frac{A_{6} B_{4}}{A_{4}} \\
& A_{1}=\frac{A_{2} B_{1}}{B_{2}} .
\end{aligned}
$$

And other parameters are arbitrary.

Case 3. The parameters are that

$$
\begin{aligned}
& a_{1}=-\frac{\sqrt{\left(-B_{4} A_{9}+A_{4} B_{9}\right)\left(-4 A_{4} B_{3} A_{9}^{2}+4 A_{3} B_{4} A_{9}^{2}-A_{9} B_{4} A_{5}^{2}+B_{9} A_{4} A_{5}^{2}\right)}}{2\left(-B_{4} A_{9}+A_{4} B_{9}\right) A_{9}}, \\
& c_{1}=-\frac{\sqrt{B_{1} B_{2}}}{B_{2}}
\end{aligned}
$$




$$
\begin{aligned}
& b_{1}=\frac{A_{6} \sqrt{\left(-B_{4} A_{9}+A_{4} B_{9}\right)\left(-4 A_{4} B_{3} A_{9}^{2}+4 A_{3} B_{4} A_{9}^{2}-A_{9} B_{4} A_{5}^{2}+B_{9} A_{4} A_{5}^{2}\right)}}{2\left(-B_{3} A_{9}+A_{3} B_{9}\right) B_{7} A_{4} A_{9}} B_{9}, \\
& a_{0}=-\frac{A_{5}}{2 A_{9}} \\
& b_{0}=-\frac{-B_{4} A_{9}+A_{4} B_{9}}{2\left(-B_{3} A_{9}+A_{3} B_{9}\right)}, \\
& \kappa=-\frac{\sqrt{\left(-B_{4} A_{9}+A_{4} B_{9}\right)\left(-4 A_{4} B_{3} A_{9}^{2}+4 A_{3} B_{4} A_{9}^{2}-A_{9} B_{4} A_{5}^{2}+B_{9} A_{4} A_{5}^{2}\right)} B_{9}}{2\left(-B_{4} A_{9}+A_{4} B_{9}\right) B_{7} A_{9}} .
\end{aligned}
$$

The constraint parameters are that

$$
\begin{aligned}
& B_{8}=-\frac{B_{4}\left(-4 A_{6}^{2} B_{9}^{2} A_{9}^{2} A_{4} B_{3}+4 A_{6}^{2} B_{9}^{2} A_{9}^{2} A_{3} B_{4}-A_{6}^{2} B_{9}^{2} A_{5}^{2} B_{4} A_{9}+A_{6}^{2} B_{9}^{3} A_{5}^{2} A_{4}+A_{4}^{2} A_{9}^{3} B_{7}^{2} B_{4}-A_{4}^{3} A_{9}^{2} B_{7}^{2} B_{9}\right)}{4 A_{4}^{2} A_{9}^{2} B_{7}^{2}\left(B_{9} A_{3}-A_{9} B_{3}\right)}, \\
& B_{5}=\frac{A_{5} B_{9}}{A_{9}}, \\
& B_{6}=\frac{A_{6} B_{4}}{A_{4}} \\
& A_{8}=-\frac{-4 A_{6}^{2} B_{9}^{2} A_{9}^{2} A_{4} B_{3}+4 A_{6}^{2} B_{9}^{2} A_{9}^{2} A_{3} B_{4}-A_{6}^{2} B_{9}^{2} A_{5}^{2} B_{4} A_{9}+A_{6}^{2} B_{9}^{3} A_{5}^{2} A_{4}+A_{4}^{2} A_{9}^{3} B_{7}^{2} B_{4}-A_{4}^{3} A_{9}^{2} B_{7}^{2} B_{9}}{4 A_{4} A_{9}^{2} B_{7}^{2}\left(B_{9} A_{3}-A_{9} B_{3}\right)}, \\
& A_{7}=\frac{A_{9} B_{7}}{B_{9}} \\
& A_{1}=\frac{A_{2} B_{1}}{B_{2}} .
\end{aligned}
$$

And other parameters are arbitrary.

Case 4. The parameters are that

$$
\begin{aligned}
& c_{1}=\frac{\sqrt{B_{1} B_{2}}}{B_{2}}, \\
& \kappa=\frac{\sqrt{\left(-B_{4} A_{9}+A_{4} B_{9}\right)\left(-4 A_{4} B_{3} A_{9}^{2}+4 A_{3} B_{4} A_{9}^{2}-A_{9} B_{4} A_{5}^{2}+B_{9} A_{4} A_{5}^{2}\right)} B_{9}}{2\left(-B_{4} A_{9}+A_{4} B_{9}\right) B_{7} A_{9}} \\
& b_{0}=-\frac{-B_{4} A_{9}+A_{4} B_{9}}{2\left(-B_{3} A_{9}+A_{3} B_{9}\right)}, \\
& a_{1}=\frac{\sqrt{\left(-B_{4} A_{9}+A_{4} B_{9}\right)\left(-4 A_{4} B_{3} A_{9}^{2}+4 A_{3} B_{4} A_{9}^{2}-A_{9} B_{4} A_{5}^{2}+B_{9} A_{4} A_{5}^{2}\right)}}{2\left(-B_{4} A_{9}+A_{4} B_{9}\right) A_{9}} \\
& a_{0}=-\frac{A_{5}}{2 A_{9}}, \\
& b_{1}=\frac{-A_{6} \sqrt{\left(-B_{4} A_{9}+A_{4} B_{9}\right)\left(-4 A_{4} B_{3} A_{9}^{2}+4 A_{3} B_{4} A_{9}^{2}-A_{9} B_{4} A_{5}^{2}+B_{9} A_{4} A_{5}^{2}\right)}}{2\left(-B_{3} A_{9}+A_{3} B_{9}\right) B_{7} A_{4} A_{9}}
\end{aligned}
$$




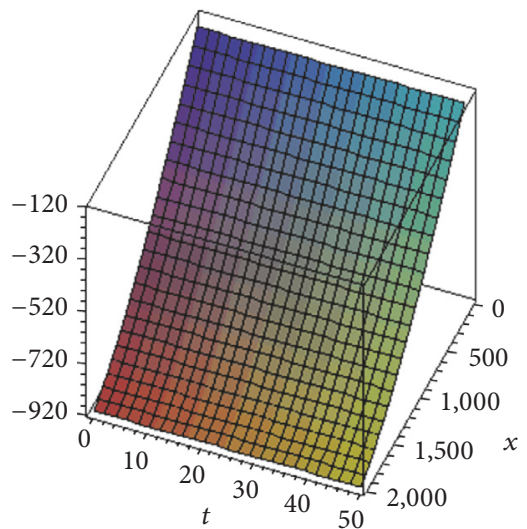

(a) Displacement of $U_{1}$

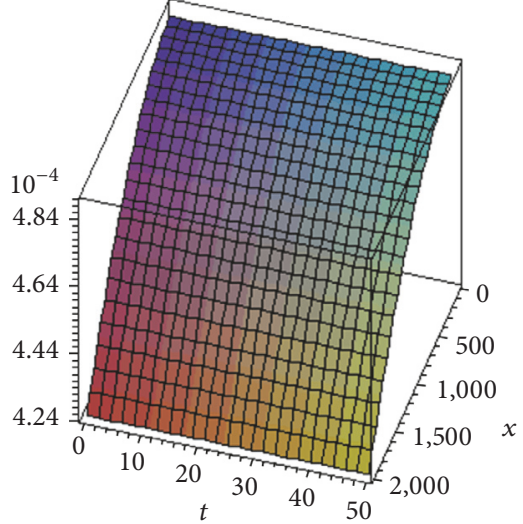

(b) Displacement of $U_{2}$

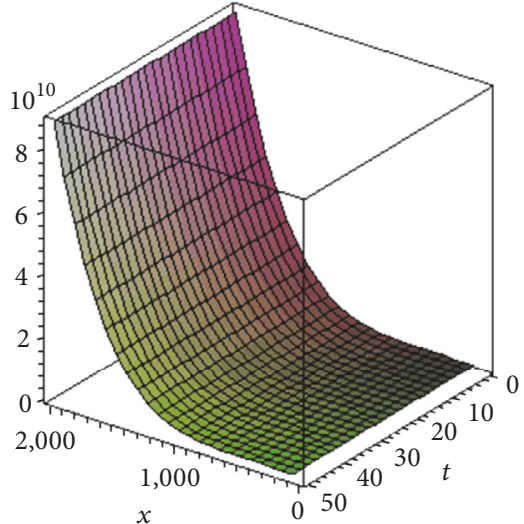

(c) Tension surface

Figure 2: The displacements and tension surfaces in Case 1.

The constraint parameters are that

$$
\begin{aligned}
& B_{8}=-\frac{B_{4}\left(-4 A_{6}^{2} B_{9}^{2} A_{9}^{2} A_{4} B_{3}+4 A_{6}^{2} B_{9}^{2} A_{9}^{2} A_{3} B_{4}-A_{6}^{2} B_{9}^{2} A_{5}^{2} B_{4} A_{9}+A_{6}^{2} B_{9}^{3} A_{5}^{2} A_{4}+A_{4}^{2} A_{9}^{3} B_{7}^{2} B_{4}-A_{4}^{3} A_{9}^{2} B_{7}^{2} B_{9}\right)}{4 A_{4}^{2} A_{9}^{2} B_{7}^{2}\left(B_{9} A_{3}-A_{9} B_{3}\right)}, \\
& B_{5}=\frac{A_{5} B_{9}}{A_{9}}, \\
& B_{6}=\frac{A_{6} B_{4}}{A_{4}} \\
& A_{8}=-\frac{-4 A_{6}^{2} B_{9}^{2} A_{9}^{2} A_{4} B_{3}+4 A_{6}^{2} B_{9}^{2} A_{9}^{2} A_{3} B_{4}-A_{6}^{2} B_{9}^{2} A_{5}^{2} B_{4} A_{9}+A_{6}^{2} B_{9}^{3} A_{5}^{2} A_{4}+A_{4}^{2} A_{9}^{3} B_{7}^{2} B_{4}-A_{4}^{3} A_{9}^{2} B_{7}^{2} B_{9}}{4 A_{4} A_{9}^{2} B_{7}^{2}\left(B_{9} A_{3}-A_{9} B_{3}\right)}, \\
& A_{7}=\frac{A_{9} B_{7}}{B_{9}}, \\
& A_{1}=\frac{A_{2} B_{1}}{B_{2}} .
\end{aligned}
$$

And other parameters are arbitrary.

To express the solutions clearly, the plots of the solutions are drawn, as shown in Figures 2-5, and the parameters used are shown in Table 1. The four solitary solutions are 2 left traveling waves and 2 right traveling waves according to the expressions of $c$. From the figures, it can be seen that, in the four solitary solutions, two of them introduce solitary tension in the system, and the tension does not change with the time delay. The other solitary solutions in $U_{1}$ and $U_{2}$ induce solitary wave tension, but with the time, the tension attenuates and disappears, and the energy is absorbed by the line and depleted by the damping from structure and external medium. The solitary tension wave in line may induce the breakage of line structure.
In the problem of taut-slack mooring line, the pretension is an important factor which relates to the line station. At the same exciting loading, when the pretension is altered, the tension curves are different, as shown in Figure 6. It can be seen that when the pretension is $10000 \mathrm{~N}$, the solution is 2-solitary solution, and with the pretension increasing, the tension changes from solitary solution to snap tension, and with the continuous increasing, the curve converts to continuous line, until straight line, which is corresponding to the taut mooring line. In the process of increasing the pretension, the mooring line transfers from slack to taut, accompanied with tension skip.

Given $P_{0}$ as $26000 \mathrm{~N}$, the tension at $s=1900 \mathrm{~m}$ is calculated as shown in Figure 7. The results show that there is a tension skip at about $t=7 \mathrm{~s}$, and the tension decreases. 


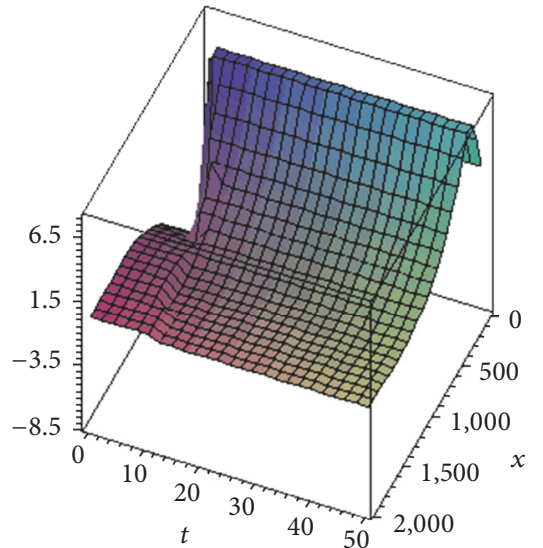

(a) Displacement of $U_{1}$

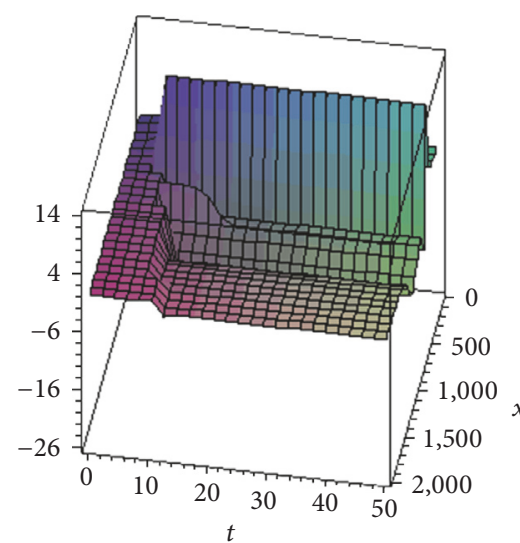

(b) Displacement of $U_{2}$

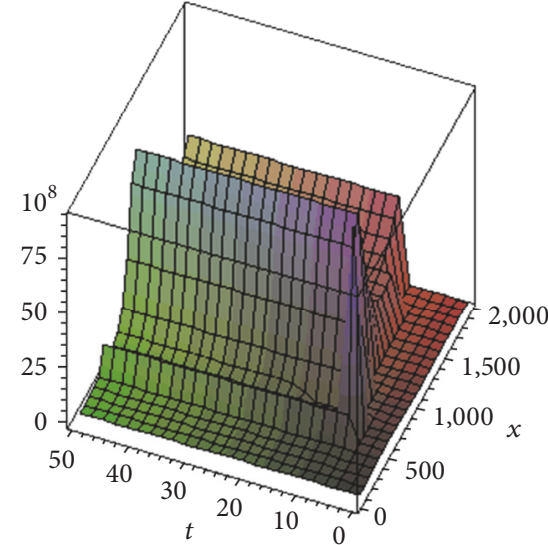

(c) Tension surface

Figure 3: The displacements and tension surfaces in Case 2.

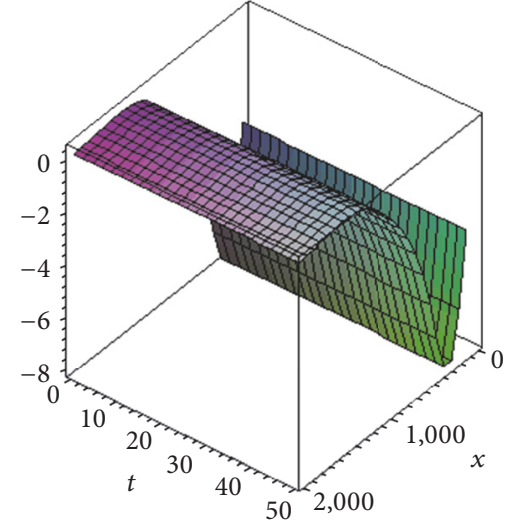

(a) Displacement of $U_{1}$

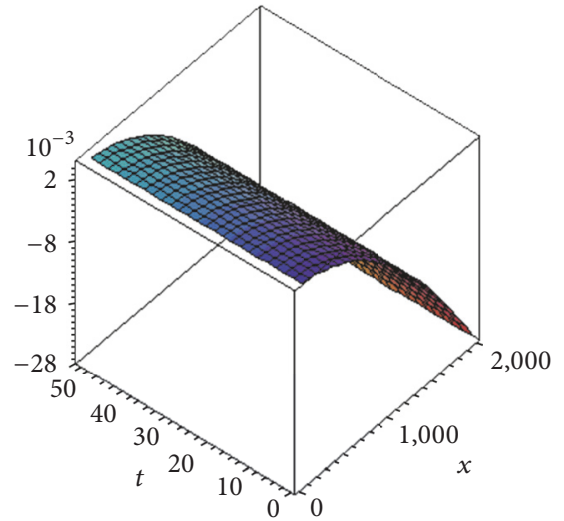

(b) Displacement of $U_{2}$

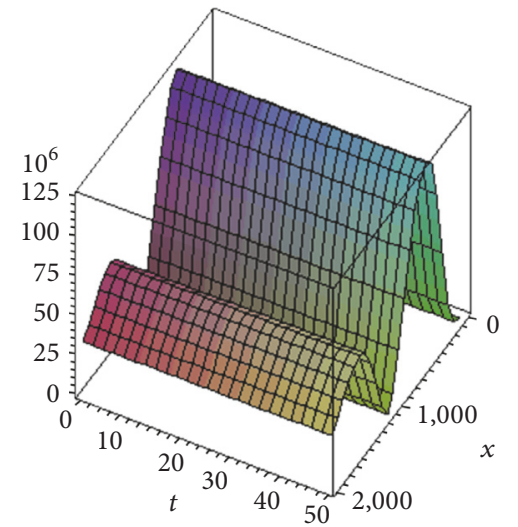

(c) Tension surface

FIgURE 4: The displacements and tension surfaces in Case 3.

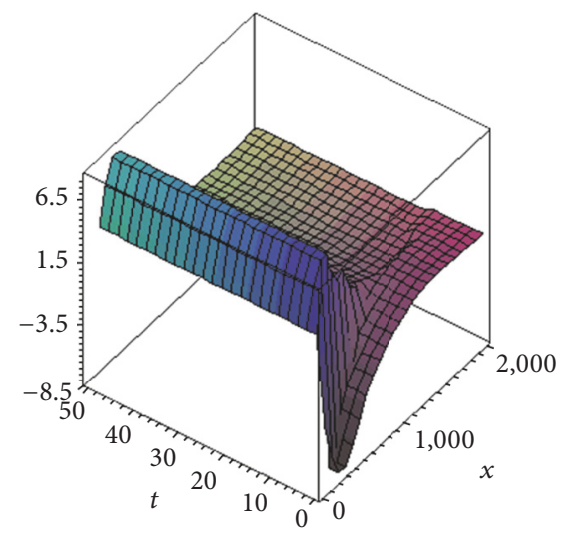

(a) Displacement of $U_{1}$

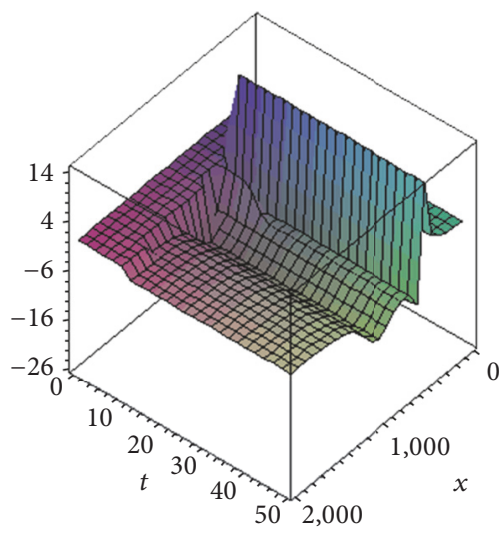

(b) Displacement of $U_{2}$

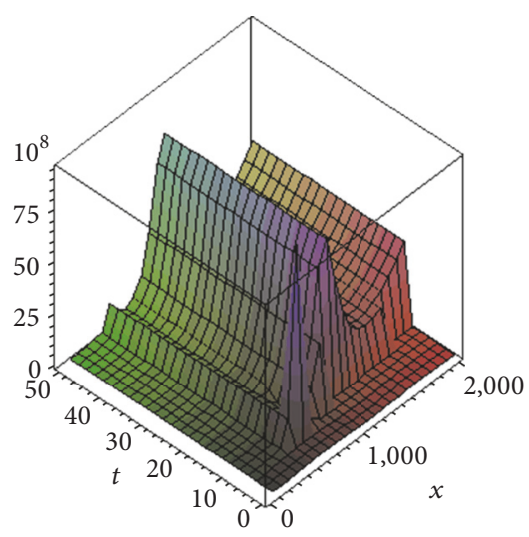

(c) Tension surface

FIgURE 5: The displacements and tension surfaces in Case 4.

When the parameters are given the same as those in [19], where the length of cable is $30 \mathrm{~m}$, excitation frequency is $1.2 \mathrm{~Hz}$, and amplitude is $8 \mathrm{~cm}$, the calculated result is shown in Figure 8, and the experimental result is shown in
Figure 9. The calculated maximum tension is $945.5 \mathrm{~N}$, and it is $790.8 \mathrm{~N}$ in the experiment. This is because the cable is elastic, and the pretension is not so accurate in the experiment. Excluding the effect, the theoretical result is consistent with 


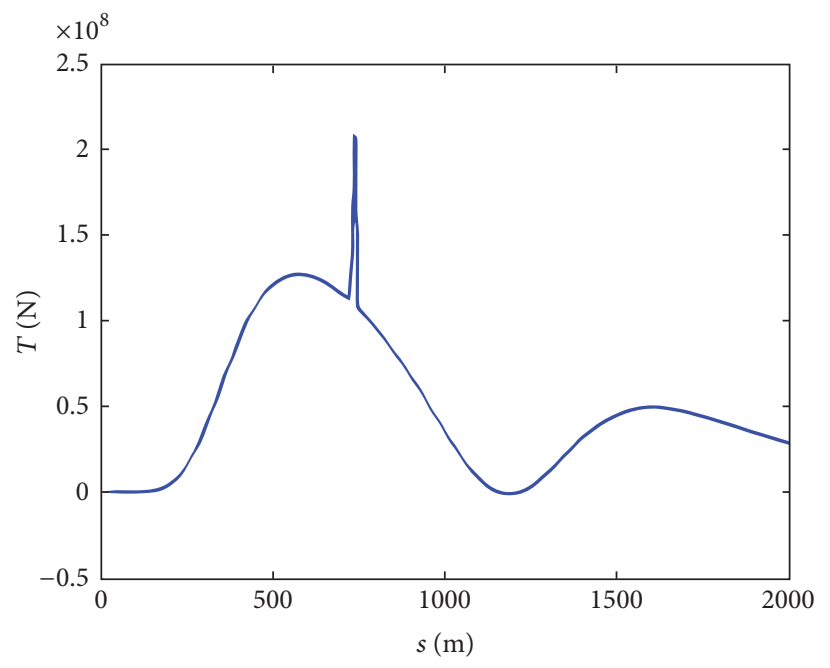

(a) $P_{0}=10000 \mathrm{~N}$

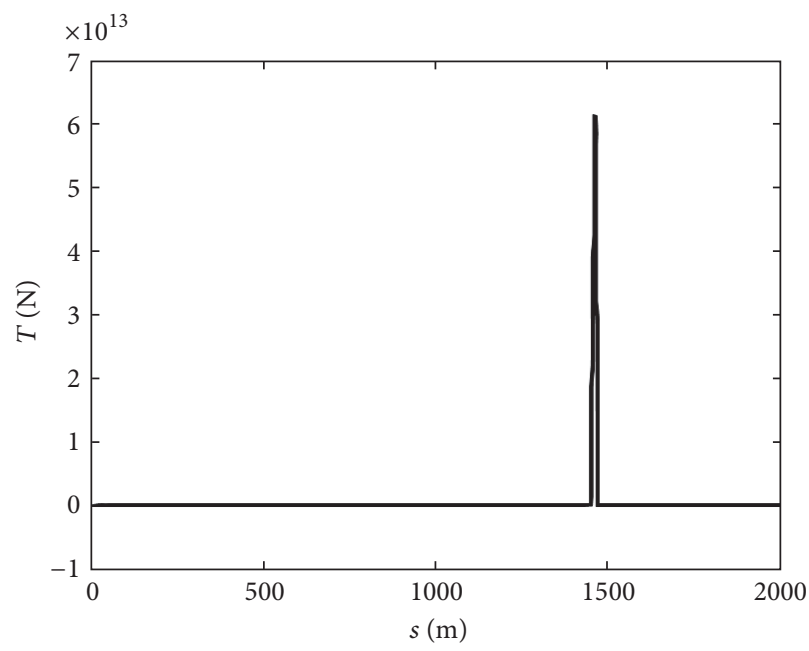

(c) $P_{0}=20000 \mathrm{~N}$

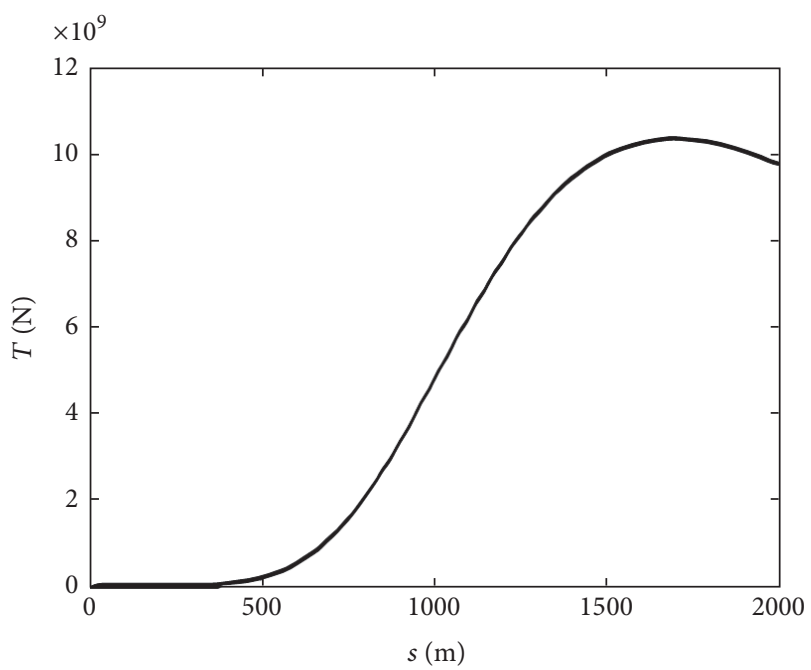

(e) $P_{0}=30000 \mathrm{~N}$

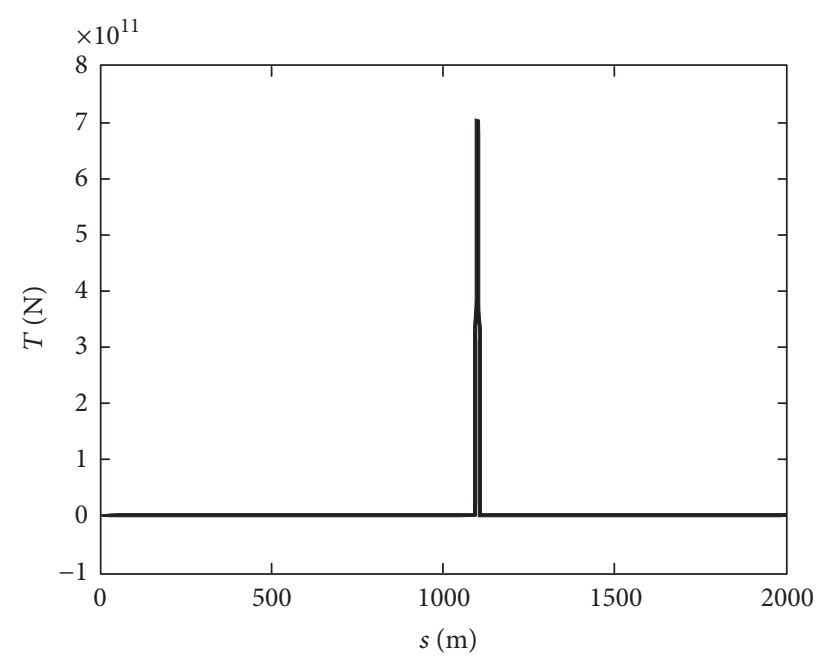

(b) $P_{0}=15000 \mathrm{~N}$

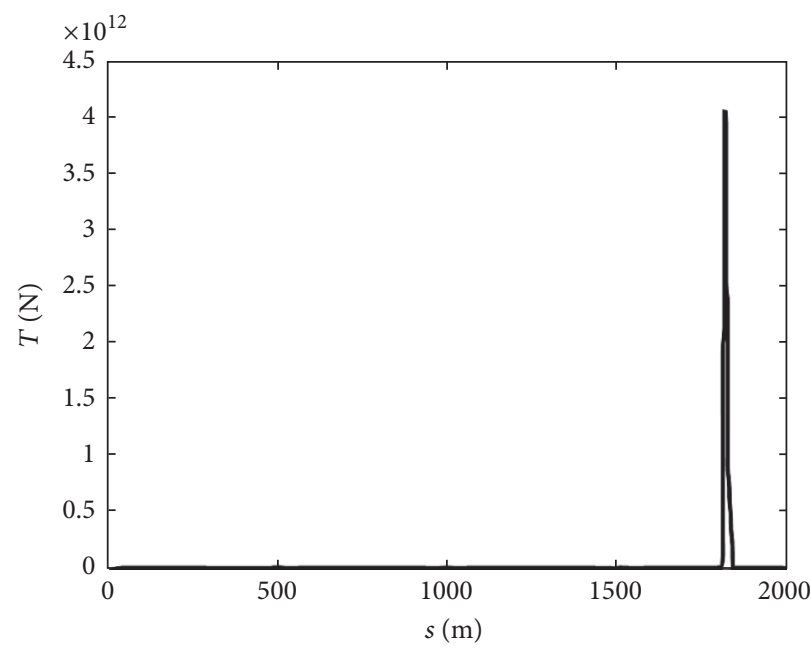

(d) $P_{0}=25000 \mathrm{~N}$

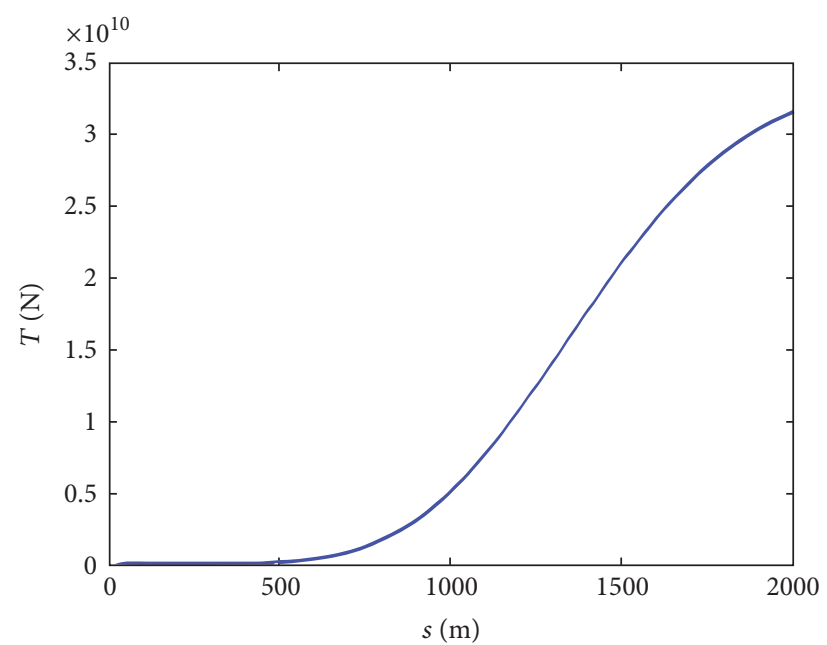

(f) $P_{0}=40000 \mathrm{~N}$

FIGURE 6: Tension curve with different pretension. 


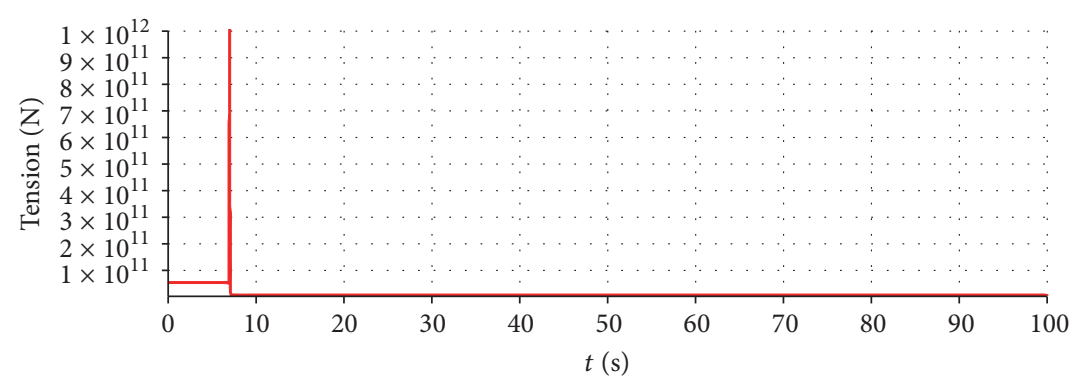

FIGURE 7: Tension history curve in mooring line at $s=1900 \mathrm{~m}$ with the method in this paper.

TABLE 1: Parameters used in the calculation.

\begin{tabular}{lcc}
\hline Quantity & Symbol & Value \\
\hline Modulus of elasticity & $E$ & $1.0 \times 10^{11} \mathrm{~Pa}$ \\
Density of cable & $\rho_{c}$ & $3750 \mathrm{~kg} / \mathrm{m}^{3}$ \\
Cross-sectional area & $A$ & $314.2 \mathrm{~mm}^{2}$ \\
Drag coefficient in normal direction & $C_{D n}$ & 1.05 \\
Inertia coefficient & $C_{m}$ & 1 \\
Exciting frequency & $\omega$ & $5 \mathrm{rad} / \mathrm{s}$ \\
Static pretension & $P_{0}$ & $27 \mathrm{kN}$ \\
Length & $L$ & $2000 \mathrm{~m}$ \\
Water density & $\rho_{w}$ & $1025 \mathrm{~kg} / \mathrm{m}^{3}$ \\
Drag coefficient in tangent direction & $C_{D t}$ & 0.01 \\
Added mass coefficients & $C_{a t} C_{a n}$ & 1 \\
Exciting amplitude & $A_{b}$ & $10 \mathrm{~m}$ \\
\hline
\end{tabular}

the experimental one. It indicates that the calculating method in this paper may be believable and feasible.

The results show that the skip tension is reduced by the system parameters, and different combination of parameters may introduce different tension in line, and the uncertainty may cause the breakage of mooring system.

\section{Conclusions}

In this paper, the continuous model is introduced, and the nonlinear partial differential equations of taut-slack mooring line system are transferred to nonlinear algebraic equations through tanh method, and four solitary solutions are obtained further. To express the results clearly, the curve surfaces of displacement $U_{1}, U_{2}$ and tension $T$ are plotted. The results show that there are four different solutions in the system. With the pretension increasing, the tension changes from solitary solution to snap tension, and when the pretension increased further, the curve converts to continuous line, until straight line, which is corresponding to the taut mooring line. In the process of increasing the pretension, the mooring line transfers from slack to taut, accompanied with tension skip, which is reduced by the system parameters, and different combination of parameters may introduce different tension in line, and the uncertainty may cause the breakage of mooring system. The results have an agreement with

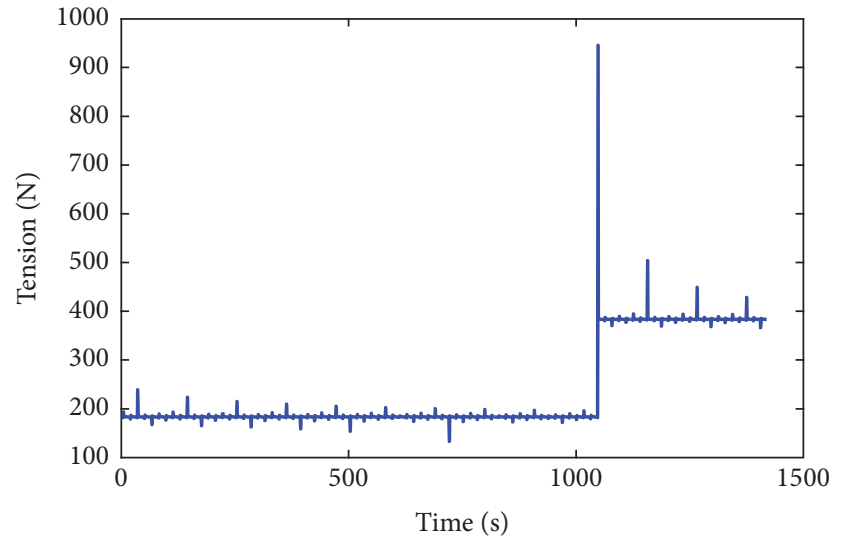

FIGURE 8: Tension history curves in mooring line simulated with the same parameters in [19].

experiment, which shows that the calculating method in this paper may be believable and feasible.

\section{Appendix}

$$
\begin{aligned}
& A_{1}=\frac{E}{\rho}+\frac{P_{0}}{\rho A}+\frac{1}{2} g \lambda s^{2} \\
& A_{2}=1+C_{a t} \rho_{w} \frac{\pi d^{2}}{4 \rho A} \\
& A_{3}=g \lambda s \\
& A_{4}=g\left(\frac{3}{2} \lambda^{3} s^{2}-\frac{1}{2} \lambda^{7} s^{6}-\lambda\right) \\
& A_{5}=\left(g \lambda^{2} s+2 g \lambda^{4} s^{3}+2 \frac{E}{\rho} \lambda^{3} s\right) \\
& A_{6}=g \lambda s \\
& A_{7}=\left(g \lambda^{2} s^{2}+\frac{E}{\rho} \lambda^{3} s^{2}+g \lambda^{4} s^{4}-\frac{E}{\rho} \lambda-2 g\right) \\
& A_{8}=2 \frac{E}{\rho}\left(\lambda^{6} s^{3}-\lambda^{4} s\right)
\end{aligned}
$$




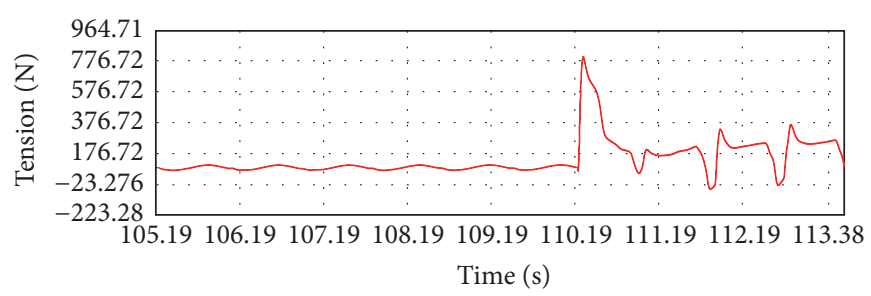

Figure 9: Tension history curves in mooring line end in middle stage at exciting end [19].

$$
\begin{aligned}
& A_{9}=6 \frac{E}{\rho}\left(\lambda^{6} s^{3}-\lambda^{4} s\right) \\
& A_{10}=\frac{E}{\rho}\left(2 \lambda^{6} s^{2}-3 \lambda^{8} s^{4}+2 \lambda^{10} s^{6}-\frac{1}{2} \lambda^{12} s^{8}-\frac{1}{2} \lambda^{4}\right) \\
& A_{11}=3 \frac{E}{\rho}\left(\lambda^{5} s-2 \lambda^{7} s^{3}+\lambda^{9} s^{5}\right) \\
& A_{12}=3 \frac{E}{\rho} \\
& A_{13}=4 \frac{E}{\rho}\left(\lambda^{2}-2 \lambda^{4} s^{2}+\lambda^{6} s^{4}\right) \\
& A_{14}=\frac{3}{2} \frac{E}{\rho} \\
& A_{15}=4 \frac{E}{\rho}\left(\lambda^{2}-2 \lambda^{4} s^{2}+\lambda^{6} s^{4}\right) \\
& A_{16}=2 \frac{E}{\rho}\left(3 \lambda^{5} s^{2}-3 \lambda^{7} s^{4}+\lambda^{9} s^{6}-\lambda^{3}\right) \\
& A_{17}=2 \frac{E}{\rho}\left(\lambda^{6} s^{3}-\lambda^{4} s\right) \\
& A_{18}=\frac{E}{\rho}\left(\frac{1}{2} \lambda^{6} s^{4}+\frac{1}{2} \lambda^{2}-\lambda^{4} s^{2}\right) \\
& A_{19}=\frac{E}{\rho} \lambda^{3} s \\
& A_{20}=\frac{E}{\rho}\left(\lambda^{3} s^{2}-\lambda\right) \\
& A_{21}=\frac{E}{\rho}\left(\lambda^{3} s^{2}-\lambda\right) \\
& A_{22}=\frac{E}{\rho}\left(\frac{1}{2} \lambda^{2}-\lambda^{4} s^{2}+\frac{1}{2} \lambda^{6} s^{4}\right) \\
& A_{23}=C_{d t} \frac{\rho_{w} d}{2 \rho A} \\
& B_{1}=\frac{1}{2} g \lambda s^{2}+\frac{P_{0}}{\rho A} \\
& B_{2}=1+C_{a n} \rho_{w} \frac{\pi d^{2}}{4 \rho A}
\end{aligned}
$$

$$
\begin{aligned}
B_{3}= & g\left(1-\lambda^{4} s^{4}-\lambda^{2} s^{2}\right) \\
B_{4}= & g\left(-\lambda^{2} s-2 \lambda^{4} s^{3}\right) \\
B_{5}= & 2 \frac{E}{\rho} \lambda^{4} s^{2}-\frac{E}{\rho} \lambda^{6} s^{4}-g \lambda-\frac{1}{2} g \lambda^{7} s^{6}+\frac{3}{2} g \lambda^{3} s^{2} \\
& -\frac{E}{\rho} \lambda^{2}
\end{aligned}
$$$$
B_{6}=2 g-\frac{E}{\rho} \lambda^{3} s^{2}-g \lambda^{4} s^{4}-g \lambda^{2} s^{2}+\frac{E}{\rho} \lambda
$$$$
B_{7}=g \lambda s
$$$$
B_{8}=\frac{1}{2} \frac{E}{\rho}\left(3 \lambda^{7} s^{4}-\lambda^{9} s^{6}-3 \lambda^{5} s^{2}+\lambda^{3}\right)
$$$$
B_{9}=\frac{3}{2} \frac{E}{\rho}\left(3 \lambda^{7} s^{4}-\lambda^{9} s^{6}-3 \lambda^{5} s^{2}+\lambda^{3}\right)
$$$$
B_{10}=3 \frac{E}{\rho}\left(-\lambda^{5} s+2 \lambda^{7} s^{3}-\lambda^{9} s^{5}\right)
$$$$
B_{11}=\frac{E}{\rho}\left(2 \lambda^{6} s^{2}-3 \lambda^{8} s^{4}+2 \lambda^{10} s^{6}-\frac{1}{2} \lambda^{12} s^{8}-\frac{1}{2} \lambda^{4}\right)
$$$$
B_{12}=\frac{5}{2} \frac{E}{\rho}\left(\lambda-\lambda^{3} s^{2}\right)
$$$$
B_{13}=\frac{1}{2} \frac{E}{\rho}\left(\lambda^{3} s^{2}-\lambda\right)
$$$$
B_{14}=\frac{E}{\rho}\left(\lambda-\lambda^{3} s^{2}\right)
$$$$
B_{15}=2 \frac{E}{\rho} \lambda^{3} s
$$$$
B_{16}=2 \frac{E}{\rho} \lambda^{3} s
$$$$
B_{17}=2 \frac{E}{\rho}\left(3 \lambda^{7} s^{4}-3 \lambda^{5} s^{2}-\lambda^{9} s^{6}+\lambda^{3}\right)
$$$$
B_{18}=2 \frac{E}{\rho}\left(\lambda^{6} s^{3}-\lambda^{4} s\right)
$$$$
B_{19}=\frac{E}{\rho} \lambda^{3} s
$$ 


$$
\begin{aligned}
& B_{20}=\frac{E}{\rho}\left(\frac{1}{2} \lambda^{6} s^{4}-\lambda^{4} s^{2}+\frac{1}{2} \lambda^{2}\right) \\
& B_{21}=\frac{E}{\rho}\left(\lambda-\lambda^{3} s^{2}\right) \\
& B_{22}=\frac{E}{\rho}\left(\lambda^{3} s^{2}-\lambda\right) \\
& B_{23}=\frac{E}{\rho}\left(\lambda-\lambda^{3} s^{2}\right) \\
& B_{24}=\frac{3}{2} \frac{E}{\rho} \\
& B_{25}=\frac{E}{\rho}\left(\frac{1}{2} \lambda^{2}-\lambda^{4} s^{2}+\frac{1}{2} \lambda^{6} s^{4}\right) \\
& B_{26}=C_{d n} \frac{\rho_{w} d}{2 \rho A} .
\end{aligned}
$$

\section{Conflicts of Interest}

The authors declare that they have no conflicts of interest.

\section{Acknowledgments}

Grateful thanks are due to the National Natural Science Foundation of China (Grant no. 51479136, no. 11502161, and no. 11302145) and Tianjin Natural Science Foundation (no. 17JCYBJC18700).

\section{References}

[1] J. Kai-hui, Study on buoy mooring system [Ph.D. thesis], Tianjin University, Tianjin, China, 2005.

[2] J. M. Niedzwecki and S. K. Thampi, "Snap loading of marine cable systems," Applied Ocean Research, vol. 13, no. 1, pp. 2-11, 1991.

[3] S. Huang and D. Vassalos, "A numerical method for predicting snap loading of marine cables," Applied Ocean Research, vol. 15, no. 4, pp. 235-242, 1993.

[4] D. Vassalos and S. Huang, "Dynamics of small-sagged taut-slack marine cables," Computers and Structures, vol. 58, no. 3, pp. 557$562,1996$.

[5] S. Huang, "Stability analysis of the heave motion of marine cable-body systems," Ocean Engineering, vol. 26, no. 6, pp. 531$546,1999$.

[6] D. Vassalos, S. Huang, and A. Kourouklis, "Experimental investigation of snap loading of marine cables," in Proceedings of the Fourteenth International Offshore and Polar Engineering Conference - ISOPE 2004, pp. 164-168, May 2004.

[7] R. Pascoal, S. Huang, N. Barltrop, and C. Guedes Soares, "Equivalent force model for the effect of mooring systems on the horizontal motions," Applied Ocean Research, vol. 27, no. 3, pp. 165-172, 2005.

[8] S.-X. Zhang, Y.-G. Tang, and X.-J. Liu, "The analytical solution of taut-slack mooring line with tanhmethod," in Proceedings of the ASME 2012 31st International Conference on Ocean, Offshore and Arctic Engineering, OMAE 2012, pp. 161-165, July 2012.
[9] A. H. Khater, W. Malfliet, D. K. Callebaut, and E. S. Kamel, "The tanh method, a simple transformation and exact analytical solutions for nonlinear reaction-diffusion equations," Chaos, Solitons \& Fractals, vol. 14, no. 3, pp. 513-522, 2002.

[10] L. Huibin and W. Kelin, "Exact solutions for two nonlinear equations," Journal of Physics A: Mathematical and General, vol. 23, no. 17, pp. 3923-3928, 1990.

[11] A.-M. Wazwaz, "The tanh method for traveling wave solutions of nonlinear equations," Applied Mathematics and Computation, vol. 154, no. 3, pp. 713-723, 2004.

[12] M. A. Abdou and A. A. Soliman, "Modified extended tanhfunction method and its application on nonlinear physical equations," Physics Letters A, vol. 353, no. 6, pp. 487-492, 2006.

[13] X. Zheng, Y. Chen, and H. Zhang, "Generalized extended tanhfunction method and its application to $(1+1)$-dimensional dispersive long wave equation," Physics Letters. A, vol. 311, no. 2-3, pp. 145-157, 2003.

[14] H. Zhi and H. Zhang, "Applications of the combined tanh function method with symmetry method to the nonlinear evolution equations," Applied Mathematics and Computation, vol. 188, no. 1, pp. 385-393, 2007.

[15] Z.-b. Li and Y.-p. Liu, "RATH: a Maple package for finding travelling solitary wave solutions to nonlinear evolution equations," Computer Physics Communications, vol. 148, no. 2, pp. 256-266, 2002.

[16] N. C. Perkins and C. D. Mote Jr., "Three-dimensional vibration of travelling elastic cables," Journal of Sound and Vibration, vol. 114 , no. 2, pp. 325-340, 1987.

[17] Y.-p. Liu and Z.-b. Li, "A Maple package for finding exact solitary wave solutions of coupled nonlinear evolution equations," Computer Physics Communications, vol. 155, no. 1, pp. 65-76, 2003.

[18] Z.-B. Li and Y.-P. Liu, "RAEEM: A Maple package for finding a series of exact traveling wave solutions for nonlinear evolution equations," Computer Physics Communications, vol. 163, no. 3, pp. 191-201, 2004.

[19] S.-X. Zhang, Y.-G. Tang, and X.-J. Liu, "Experimental investigation of nonlinear dynamic tension in mooring lines," Journal of Marine Science and Technology, vol. 17, no. 2, pp. 181-186, 2012. 


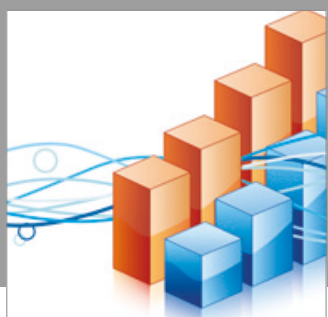

Advances in

Operations Research

vatersals

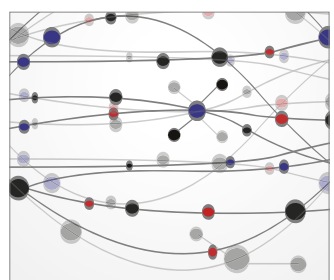

\section{The Scientific} World Journal
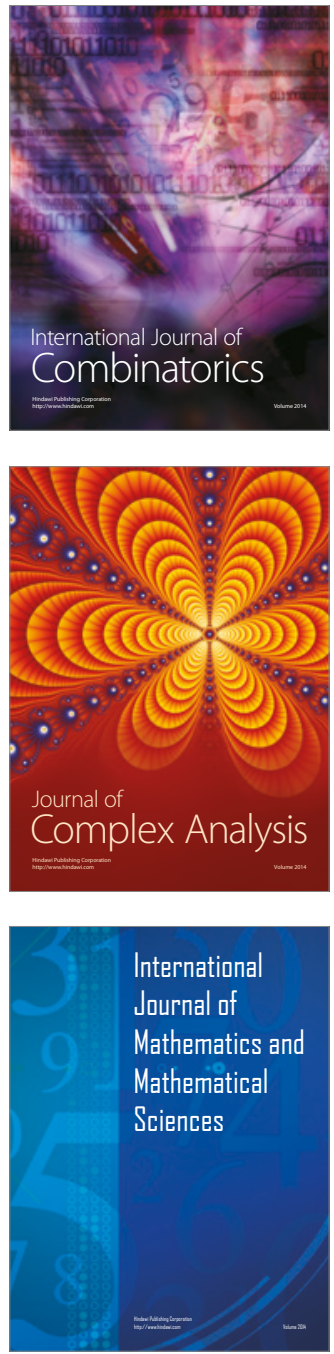
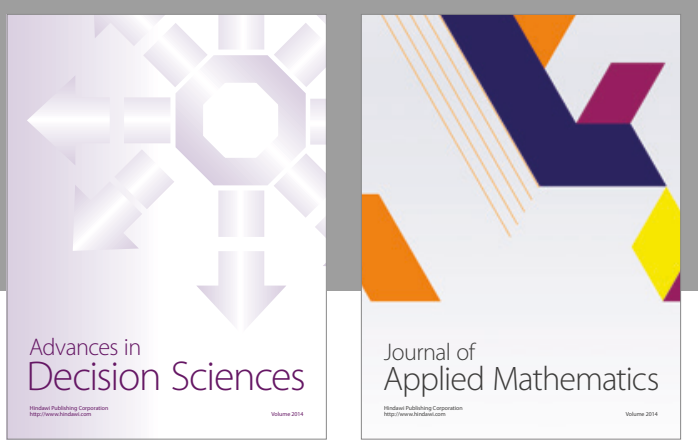

Algebra

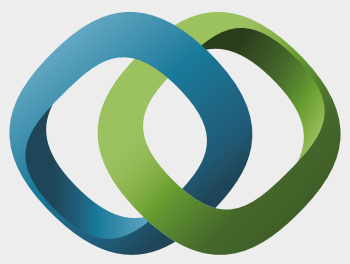

\section{Hindawi}

Submit your manuscripts at

https://www.hindawi.com
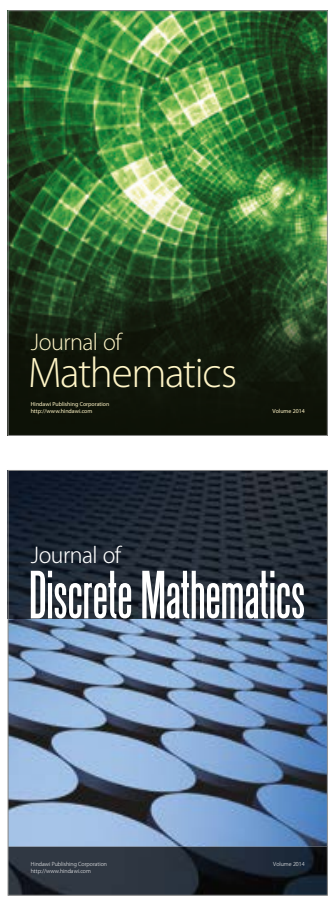

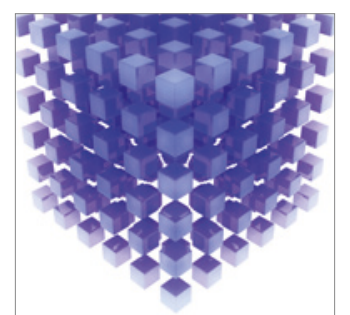

Mathematical Problems in Engineering
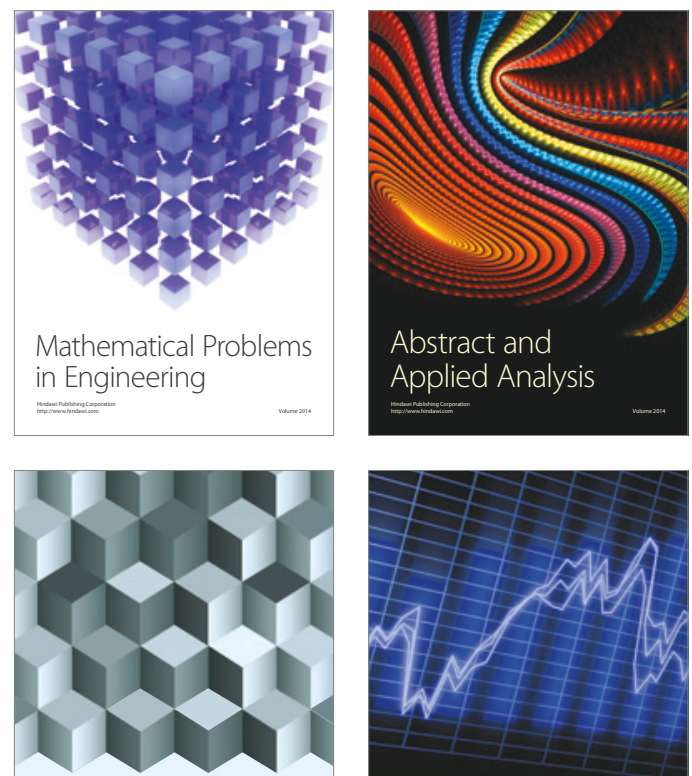

Journal of

Function Spaces

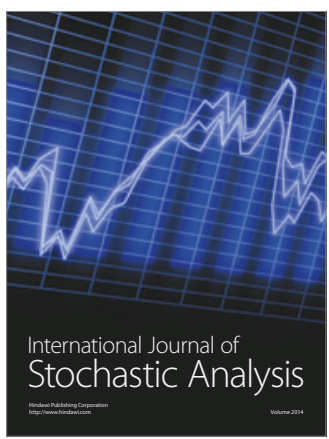

Probability and Statistics
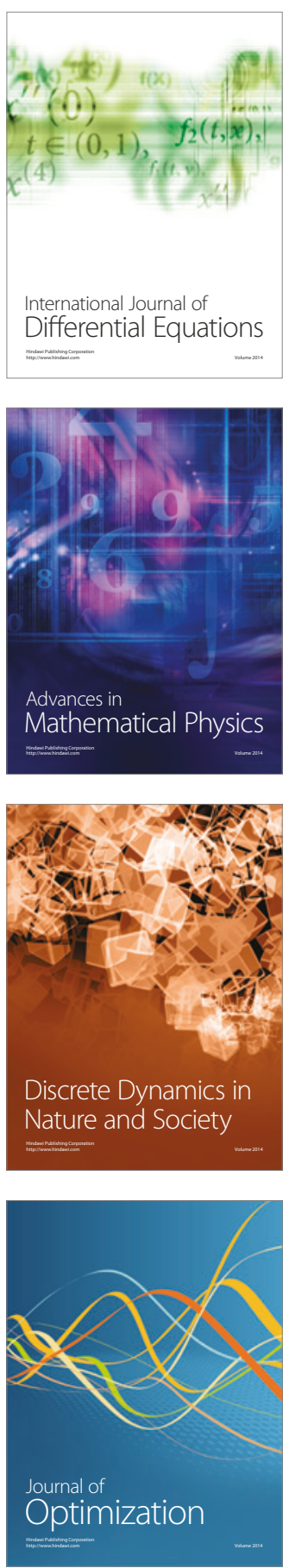